\title{
Change is good: life outside the nucleus
}

\section{Randy Schekman}

No one knows what the future holds, but one thing is clear, everything changes. The best advice for a young scholar is to remain flexible and seek new experiences, unbound by past successes or failures. The temptation is to stick with what works, but to be independent in the research world, change is good.

I was ignorant of this view when I began my research career as an undergraduate student at the University of California, Los Angeles. Fate brought me in touch with a new assistant professor, Dan Ray of the then Zoology Department. During this period, I developed an interest in the replicative mechanism of M13 and $\phi X 174$ phage DNA, a field that was essentially created by the pioneering biophysicist Robert Sinsheimer at the California Institute of Technology. The burning question at the time was how a single-stranded circular DNA template could be copied to a double-stranded replicative form, and then how this replicative form could spool-out new single-stranded circular progeny. The analytic tool was genetics alongside density and velocity gradient separation of replicative intermediates.

At the same time, using a classic enzymological approach, Arthur Kornberg and his postdoctoral fellow Mehran Goulian had succeeded in converting $\phi$ X174 single-stranded circles to a double-stranded form using Escherichia coli DNA polymerase (now called polymerase I) and a crude source of DNA oligonucleotide primers obtained by heating an E. coli lysate.

I was convinced that this sort of biochemical approach would eventually clarify the mechanism of DNA replication. I had the

Randy Schekman is Professor of Cell and Developmental Biology in the Department of Molecular and Cell Biology, University of California at Berkeley, Berkeley, CA 94720-3200, USA.

e-mail: schekman@berkeley.edu great fortune of becoming Kornberg's graduate student; thus making the first important change in my career - from using a primarily in vivo to an in vitro approach to study a complex cellular process.

In Kornberg's laboratory, I teamed up with Doug Brutlag and Bill Wickner to develop a method to define the physiologically relevant dna gene products essential for conversion of $\phi X 174$ single stranded DNA to the replicative form. We succeeded in producing a soluble $E$. coli lysate that was competent for replication, but which was defective when replication mutant cells were used as a source of cytosol. This observation permitted us to isolate functional forms of several of the replication proteins, including an RNA primase and the DNA polymerase III holoenzyme, and it led Kornberg and, in a parallel effort, Jerard Hurwitz to claim success several years later by completely reconstituting replication with pure proteins and a DNA template.

I was powerfully impressed by this successful model of how to approach a problem, but put off by the fierce competition in the field of DNA replication at the time. Although my training and inclination had prepared me for a career in nucleic acid research, I was drawn by the allure of an emerging area of research in cellular membranes.

In the early 1970s, membrane cell biology remained in a descriptive phase. George Palade and his colleagues had brilliantly charted the path of secretion in pancreatic exocrine cells, yet mechanistic aspects of this process remained shrouded. At the same time, the first biochemical approaches to synthesize secretory proteins and reconstitute protein translocation in a cell-free reaction emerged in the laboratories of Blobel, Milstein and Sabatini. Other developments in the structure of native membranes led S. J. Singer and Nicholson to propose a fluid mosaic model with lipids and membrane proteins free to diffuse laterally within the plane of the bilayer.

In another corner of the cell biology world, regulation of cell division yielded to an elegant genetic dissection developed by Lee Hartwell. If such a classic approach could illuminate the genes and proteins that govern progress through the cell cycle, then surely a similar effort could help open the secretory pathway to functional analysis. And combining classical genetics with biochemical reconstitution could perhaps lead to mechanistic insights in an area as complicated as membrane assembly, as it had in DNA replication and bacteriophage particle assembly even earlier. The opportunities presented by yeast as a model eukaryote and the nascent area of membrane assembly were sufficiently alluring to encourage me to make a clean break from nucleic acids.

What better time to make a change than after an intense period of training under the influence of a powerful mentor. I jumped ship to mammalian membranes during a two-year postdoctoral stint with S. J. Singer, and then again to yeast genetics and membrane biochemistry when I moved to Berkeley to begin my independent career in 1976. Of course, such change is not without risk; my first NIH grant proposal to work on yeast membranes was trashed because I had no relevant experience or preliminary data. But with great students and colleagues at Berkeley, my laboratory and those of several former students succeeded in defining the secretory pathway with genetics and then by reconstituting and purifying many components required for vesicular traffic. I have never regretted the change. 